\title{
The Necessity and the Scheme of Super Cloud and Super Cloud Language Existence
}

\author{
SiqinQimuge \\ Department of Computer Science and Technology \\ Chifeng University \\ Chifeng 024000, China \\ Tel: 86-1860-476-0007 E-mail: siqin1848@sina.com
}

\begin{abstract}
Chunsheng Zhang
Inner Mongolia University for Nationalities College of computer science and technology

Inner Mongolia Tongliao 028043, China

Tel: 86-0475-831-3358 E-mail: zhangcs_817@sina.com.cn
\end{abstract}

Received: July 18, 2011

Accepted: August 3, 2011

doi:10.5539/cis.v4n5p92

\begin{abstract}
The technology of cloud computing has attracted wide attention since its beginning, and it will become the mainstream in IT industry of next step. However, the future situation of cloud computing technology is still in initial stage, because the technology of cloud computing is burgeoning. So the conception of super cloud and super cloud language is proposed by referring to the development route of database technology and data mining technology, and the necessity of super cloud and super cloud language existence is discussed and the scheme is given which will become the goal of the development of cloud in the future.
\end{abstract}

Keywords: Super cloud, Super cloud language, Necessity, Cloud computing, Scheme

\section{Introduction}

The concept of cloud computing rise in 1999, which is a super calculation model based on internet, and thousand of computers and servers are connected to form a computer cloud in remote data center. Therefore, cloud computing can even experience the operation ability of ten teraflops, and the strong computing capability possessed can estimate nuclear explosion, prediction of climate change and market development trend. Users can be accessed data center by computer, notebook PC, mobile phone and so on, and can operate according to user requirements.

Cloud computing is the development of distributed processing, parallel processing and grid-computing, or it is the commercial realization of these computer science concepts.

Cloud computing has infinite possible, such as safety, convenience and data sharing, and it provides the infinitely more possible (ZHANG Jian xun, 2010; ZHONG ZHisui, 2010; China Cloud Computing Network, 2010; eNet Silicon Valley Dynamic, 2010; Science and Technology Daily, 2009; Allan Alter, 2010) for using internet.

IDC called: cloud computing will be throughout the World of IT market, and our life will be changed greatly by cloud computing (Server On-Line, 2008; ZHANG Fubo, 2010; YING Shi, 2010; WANG Jiajun, 2010).

However, the future world is often not a pure cloud application of one aspect. A user may need simultaneously to integrate multiple different cloud computing services, which is an inevitable trend in the development of cloud computing. As ODBC of present database technology can connect simultaneously most kinds of heterogeneous database and data sets can include different heterogeneous data, cloud computing can facility user use (WANG E, 2009; KUANG Shenghui, 2010; HE Qing, 2010).

Therefore, the concept of super cloud is proposed in the article, which can provide numerous cloud services to users by opening cloud connection, and in terms of use's viewpoint cloud service is treated in isomorphic and is used by a standard "super cloud language" in order to achieve the goal of user requirement. 


\section{The concept of super cloud}

\subsection{Existence necessity of super cloud}

Users' demand for cloud is often not a pure, and it is generally that multiple cloud computing services are requested at the same time. For example, we need two cloud services at lease when we want to know what domestic different magazines to article type (including knowledge points, authors, fund, reference, etc ) published have latent rules.

\section{(1) Collect information of magazines}

We can collect enough articles and information of magazines by current periodical database (for instance CNKI), and pre-treats them. But data mining services do not always be provided simultaneously by these database vendors.

\section{(2) Data mining}

Based on data obtained, data mining for these data is implemented by cloud computing service.

Similar situation is more frequently, a task of users needs simultaneous multiple cloud services to complete.

Every cloud service in task exists in temporal sequence, and how to fuse reasonably these operations together. As compiling a general program, task-performing is the goal we are pursuing.

Certainly, a task can often be completed by requesting multiple cloud services many times in practical application, meanwhile, there are differences in data organization structure, data description and interactive language. If the concept of super cloud and cloud language that leaps the concrete cloud service is used, users can compile a program to perform requirements of multiple cloud services, and easily realize cloud services, which is the ultimate goal of users.

\subsection{Super cloud architecture}

The system structure of super cloud is composed up eight layers, in where user-oriented part includes five layers, which is shown in Figure 1. and the first layer namely cloud service is also decomposed into four layers.

The first layer: cloud computing service layer, expresses multiple cloud services that comes from internet.

The second layer: cloud connection layer, users can connect with cloud service by constructing the open cloud connection interface, and the convert service of general super cloud language and local cloud service language is realized.

The third layer: super cloud explanation system layer, which will transform standard super cloud language into the acceptable instructions of "super cloud connection layer".

The Fourth layer: application programs layer, which is certain application according to users and the application programs are compiled by using the standard super cloud language.

The fifth layer: user layer, which expresses various users of using application programs.

The technology level of cloud service includes four layers, as shown in Figure 2.

From cloud service angle, it consists of four parts: physical resource, virtual resource, middleware management part and service interface.

Service interface: variety norms and various standards are unified regulations on using computer in the cloud computing age, and the entrance of interactive operation in user end and cloud end can complete user or service registry, service customization and service use.

Service management middleware: middleware is located in between service and server cluster in the technology of cloud computing, and provides management and service namely the management system in system structure of cloud computing. Services, including mark, authentication, authorization, directory, safety and etc, are standardized and operated, the unified standard program interface and protocol are provided to users, and the heterogeneous nature of bottom hardware, operation system and network is hided and network resource is unified management. Its user management includes user authentication, user admission and user admission. Resource management includes load balancing, resource monitoring, fault detection and etc. Safety management includes authentication, access authorization, security audit, comprehensive protection and etc. Image management includes image construction, deployment, management and etc.

Virtual resource: is refers to some resource that can realize certain operation and certain function, but it is virtual, but not real. Such as computing pool, storage pool, network pool, database resource, and etc. Relevant virtual 
functions, including virtual environments, virtual system and virtual platform, are realized by software technology.

Physical resource: is mainly refers to some hardware equipments and technology that can support the normal operation of computer, and the equipments such as the cheap PCs, and expensive price server and disk array and so on. A cluster used in the cloud operation of computing and storage, is composed up computers separated by existing network technology, parallel technology and distributed technology, and it can provide powerful functions.

The model construction of super cloud makes the architecture of the whole super model is divided into eight layers. As compiling general programs, users have been written out application programs which applied to multiple cloud services using the standard super cloud language. While use is considered, multiple cloud services are used.

\section{Super cloud language}

The application of super cloud must have super cloud language. The following is given a discussion on the structure of super cloud language that consists of three parts in the main.

\subsection{Application interface}

Firstly, the concept of cloud object (CO) is constructed in order to realize connection to cloud computing service, and cloud object can provide serials of attributes, methods and events, and realizes service request on the cloud.

Wanting to realize the cloud computing service must create firstly the cloud object, and connection to cloud is realized by setting the attributes for cloud object.

For instance, connection to cloud is realized by setting username, password, connection parameters, returning parameters and etc.

\subsection{The basic elements of general language}

As common language, the basic elements of language include:

(1) The requirements of the style for language

(2) Data type, including simple type, coincidence type, object type and etc.

(3) Specification statement, including variable declaration, constant declaration and etc.

(4) Structural control statement, including sequential statements, branch statements, loop statement, jump statements and etc.

(5) Function, it is absolutely necessary element of language. Defining massive functions will enrich functions of language and simplify the complexity of compiling program.

\subsection{The professional cloud tool language}

Different cloud services have its own different characteristics, a cloud language of oriented different cloud services, representation methods and access mode of data in cloud are provided from the higher level, and the difference of different cloud services is balanced. According to various cloud services, accessing different cloud services is realized on the basis of cloud object (CO).

For example, concerning data mining services, cloud object includes: construction and representation of cloud dataset, dataset accessing method, data preprocessing method, data mining method, show of results, storage of results, etc.

\section{Application example}

Suppose that user tries to understand what latent rules domestic different magazines to article type (including knowledge points, authors, fund, reference and etc ) published have, the collection and compilation of data is realized by accessing cloud service of URL_1 and data mining is realized by accessing cloud service of URL_2.
dataset ds as dataset
//define data set, and record the result of query
create co_1 as cloud object
//create cloud object1
co_1.url=URL_1
co_1.name $=\mathrm{a}$
//address of cloud object1
co_1.pass $=$ p_a
//user name of cloud object1
//password of cloud object1
//other parameters of cloud object1




\begin{tabular}{|c|c|}
\hline co_1.connect & // connect cloud object1 \\
\hline create co_2 as cloud object & //create cloud object 2 \\
\hline co_2.url=URL_2 & // address of cloud object2 \\
\hline co_2.name $=\mathrm{b}$ & // user name of cloud object 2 \\
\hline \multirow[t]{2}{*}{ co_2.pass $=$ p_b } & // password of cloud object 2 \\
\hline & // other parameters of cloud object 2 \\
\hline co_2.connect & // connect cloud object2 \\
\hline megbox ("begin to query data") & // prompt to begin query \\
\hline \multicolumn{2}{|c|}{ ds =co_1.select ("output list","query condition","order by") //query, the results ex } \\
\hline megbox ("start data mining") & //prompt, start data mining \\
\hline co_2.dataset $=\mathrm{ds}$ & //data set of mining is set to ds \\
\hline co_2.dm_support $=0.7$ & //the support is set to 0.7 \\
\hline co_2.dm_confidence $=0.8$ & $/ /$ the confidence is set to 0.8 \\
\hline co_2.dm_method=apriori & $/ /$ the method of mining is set to apriori \\
\hline co_2.disp & //show the result of mining \\
\hline
\end{tabular}

\section{Prospects}

Since cloud computing has rise in 1999, it has attracted wide attention in IT fields of worldwide, and there is no unified answers in the role, the connotation and the future development of cloud computing. But the present manufacturers such as google, IBM and etc, including some domestic enterprises as china mobile-corporation have already given a trial. "China cloud computing" has been held two times. There is unquestionable that future world is the world of cloud.

As other new technology is formed, cloud computing technology now is also in fuzzy stage of the concept and the future development direction. Some presumptions of future development of cloud computing technology are proposed by referring to the development course of database technology and data mining technology and basis for the development law in the article, namely the construction scheme of super cloud and super cloud language, which is studied by the people of the same profession.

\section{References}

Allan Alter. (2010). Investigation Report on China Cloud Computing. [Online] Available: http://www.ciecloud.org/2010.

China Cloud Computing Network. (2010). What is Cloud Computing. [Online] Available: http://www.cloudcomputing-china.cn/.

eNet Silicon Valley Dynamic. (2010). China "Cloud" Provides Scientific Research Motivation for University. [Online] Available: http://www.cloudcomputing-china.cn/.

HE Qing. (2010). Massive Data Mining Based on Cloud Computing. [Online] Available: http://www.ciecloud.org/2010.

KUANG Shenghui \& LI Bo. (2010). Architecture and Application Example Analysis of Cloud Computing. Computer and Digital Engineering, 38(3), 60-63.

Science and Technology Daily. (2009). Future Information Society is in "Cloud". [Online] Available: http://www.cloudcomputing-china.cn/.

Server On-Line. (2008). IDC Called: cloud computing will be throughout the World of IT market after five years. [Online] Available: http://www.cloudcomputing-china.cn/.

WANG E \& LI Ming. (2009). The Research of Massive Data Mining Based on Cloud Computing. Modern Computer, 11, 22-25.

WANG Jiajun, LIU Zhihui, WU Jie, et al. (2010). The Development Analysis and Discussion on Application of Cloud Computing Technology. Computer Engineering and Design, 31(20), 4404-4409.

WANG Peng \& HAN Jingyi. (2009). A Study on Realizing Method in Architecture of Cloud Computing. Computer Engineering and Science, 31(A1), 11-13. 
WANG Peng. (2009). Close to Cloud Computing. Beijing: Posts and Telecommunications Press, 2009.6

YING Shi. (2010). Software Development in the Age of Cloud Computin. [Online] Available: http://www.ciecloud.org/2010.

ZHANG Fubo. (2010). Prospect on Technology and Architecture of Cloud Computing. [Online] Available: http://www.ciecloud.org/2010.

ZHANG Jian xun, GUO Zhiming \& ZHENG Chao. (2010). Review on Some Research Advances in Cloud Computing. Computer Application Research, 27(2), 429-433.

ZHONG ZHisui. (2010). The Present and Future of Cloud Computing. Modern Computer, 1, 34-37.

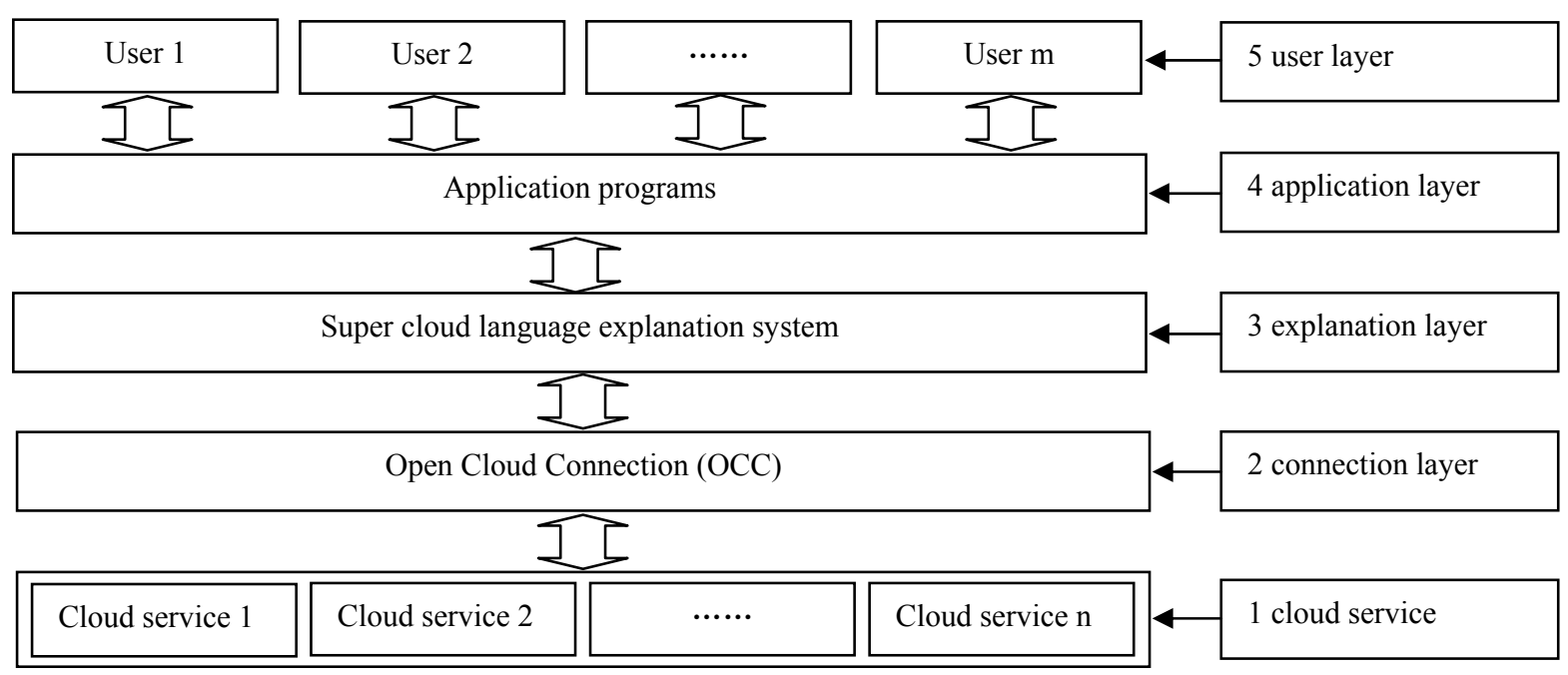

Figure 1. The User-Oriented Super Architecture

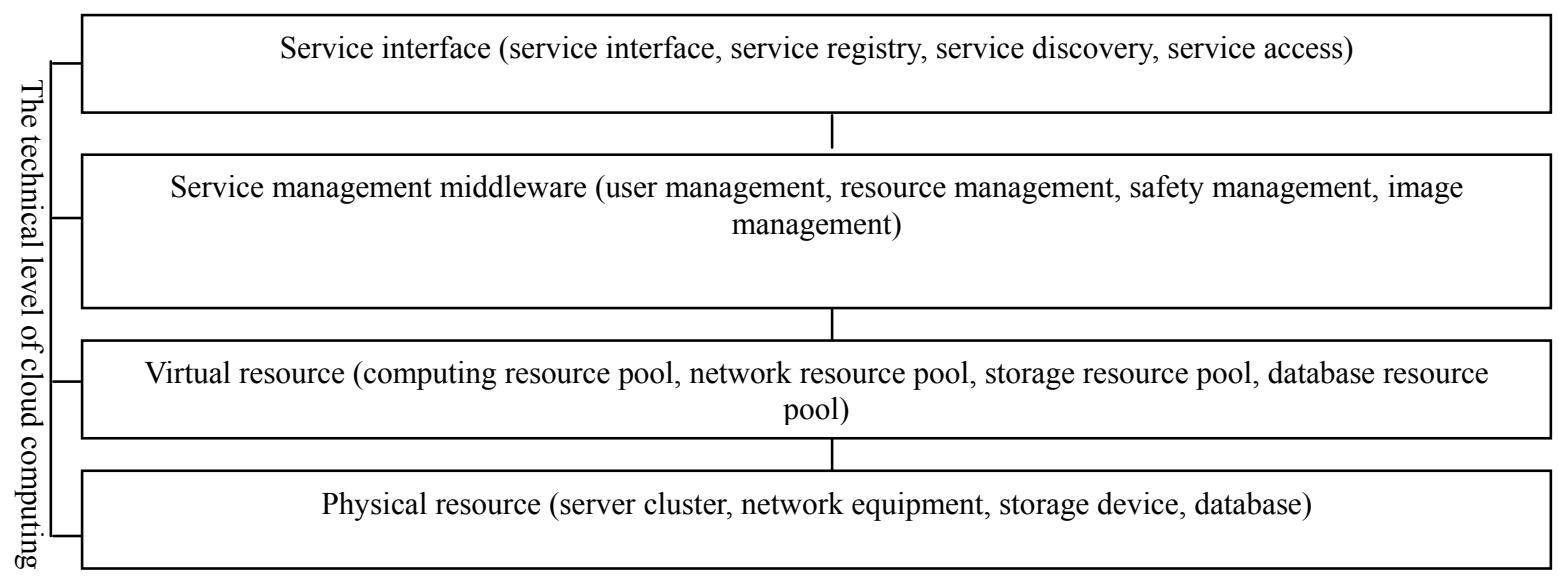

Figure 2. Part Super Architecture of Cloud Service 